University of South Carolina

Scholar Commons

4-15-2009

\title{
Frequency Doubling and Memory Effects in the Spin Hall Effect
}

Yuriy V. Pershin Dr

University of South Carolina - Columbia, pershin@physics.sc.edu

Massimiliano Di Ventra

Follow this and additional works at: https://scholarcommons.sc.edu/phys_facpub

Part of the Physics Commons

\section{Publication Info}

Published in Physical Review B, ed. Gene D. Sprouse, Volume 79, Issue 15, 2009, pages

153307-1-153307-4.

Pershin, Y. V., \& Di Ventra, M. (2009). Frequency doubling and memory effects in the spin Hall effect. Physical Review B, 79(15), 153307-1 - 153307-4. DOI: 10.1103/PhysRevb.79.153307

(C) Physical Review B, 2009, American Physical Society

This Article is brought to you by the Physics and Astronomy, Department of at Scholar Commons. It has been accepted for inclusion in Faculty Publications by an authorized administrator of Scholar Commons. For more information, please contact digres@mailbox.sc.edu. 


\title{
Frequency doubling and memory effects in the spin Hall effect
}

\author{
Yuriy V. Pershin* \\ Department of Physics and Astronomy and USC Nanocenter, University of South Carolina, Columbia, South Carolina 29208, USA \\ Massimiliano Di Ventra ${ }^{\dagger}$ \\ Department of Physics, University of California, San Diego, La Jolla, California 92093-0319, USA
}

(Received 25 February 2009; published 29 April 2009)

\begin{abstract}
We predict that when an alternating voltage is applied to a semiconducting system with inhomogeneous electron density in the direction perpendicular to main current flow, the spin Hall effect results in a transverse voltage containing a double-frequency component. We also demonstrate that there is a phase shift between applied and transverse-voltage oscillations, related to the general memristive behavior of semiconductor spintronic systems. A different method to achieve frequency doubling based on the inverse spin Hall effect is also discussed.
\end{abstract}

DOI: 10.1103/PhysRevB.79.153307

PACS number(s): 72.25.Dc, 71.70.Ej

\section{INTRODUCTION}

In optics, frequency doubling (also called second harmonic generation) is obtained from nonlinear processes, in which the frequency of photons interacting with a nonlinear material is doubled. This phenomenon was first observed in $1961^{1}$ and has found numerous applications in diverse areas of science and engineering. ${ }^{2}$ Physically, the fundamental (pump) wave propagating through a crystal with $\chi^{(2)}$ nonlinearity (due to the lack of inversion symmetry) generates a nonlinear polarization which oscillates with twice the fundamental frequency radiating an electromagnetic field with this doubled frequency. In electronics, frequency doubling is a fundamental operation for both analog and digital systems which is however achieved via complex circuits made of both passive and active circuit elements. ${ }^{3}$

As we demonstrate in this Brief Report, the possibility of generating frequency doubling need not be limited to optical processes in crystals or require complex circuits. In fact, we show it can be realized via a completely different physical mechanism using the spin Hall effect. ${ }^{4}$ Our idea is to use a material with inhomogeneous doping in the direction perpendicular to the main current flow. As it was recently shown, ${ }^{5}$ a dc voltage applied to such a system results in a transverse voltage, similar to the Hall voltage, but with a different symmetry: the sign of the transverse voltage due to the spin Hall effect does not depend on the polarity of the applied field. Therefore, when an ac voltage is applied, the transverse voltage oscillations are similar in the positive and negative halfperiods of the applied voltage, which results in the frequency doubling.

Moreover, the transverse voltage oscillations show hysteretic behavior at different frequencies. This result is reminiscent of the recent experimental demonstration of memoryresistive (memristive) behavior in certain nanoscale systems $^{6,7}$ and is consistent with our suggestion ${ }^{8}$ that some semiconductor spintronic systems are intrinsically memristive systems. ${ }^{9}$ When a time-dependent voltage is applied to such systems, their response is delayed because the adjustment of spin polarization to changing driving field requires some time (due to spin relaxation and diffusion processes). In other words, the electron spin polarization has a short- time memory on its previous state. ${ }^{8}$ A unique feature of the system investigated in this work is that effects of spin memory manifest themselves in the voltage response, while in the previous study ${ }^{8}$ spin memory effects were predicted in the current. Below, we study the frequency doubling and manifestation of spin memristive effects both analytically and numerically. This work reveals fundamental aspects of the spin Hall effect that have not been explored yet, as well as its possible use in electronic circuits.

Figure 1 shows a possible experimental setup which can be used to observe frequency doubling by using the spin Hall effect. An alternating voltage $V(t)=V_{0} \cos (\omega t)$ of frequency $\omega$ and amplitude $V_{0}$ is applied along a sample of semiconductor material (we call this $x$ direction). The electron density in the semiconductor is inhomogeneous in the direction (y) perpendicular to main current flow. As the densities of transverse (spin Hall) currents are stronger in the areas of higher electron density, transverse charge currents at a boundary between areas with different doping levels are not compensated and charge accumulation, in addition to spin accumulation, develops. ${ }^{5}$ Therefore, a transverse timedependent voltage $V_{T}$ develops between the sample boundaries $y=0$ and $y=L$, where $L$ is the sample width. According to our previous calculations ${ }^{5}$ and recent experimental results, ${ }^{10}$ it is expected that the transverse voltage oscillation amplitude is a nonlinear function of $V_{0}$.

To study this system, we employ a self-consistent twocomponent drift-diffusion model ${ }^{11,12}$ which is appropriate for

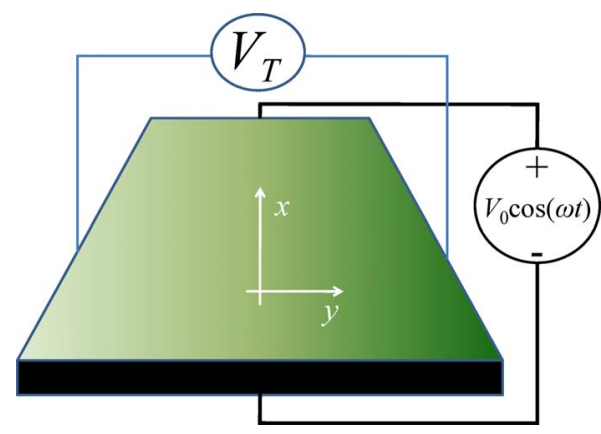

FIG. 1. (Color online) Schematics of the experimental setup. Inhomogeneous charge density is represented via a color gradient. 
the description of the extrinsic spin Hall effect, assuming that the system is macroscopic in the $x-y$ directions. The inhomogeneous charge-density profile $n(y)$ is defined via an assigned positive background density profile $N(y)$ (such as the one shown in Fig. 1) which can be obtained in different ways including inhomogeneous doping, variation of sample height or gate-induced variation of electron density. Assuming homogeneous charge and current densities in the $x$ direction and homogeneous $x$ component of the electric field in both $x$ and $y$ directions, the set of equations to be solved is

$$
\begin{gathered}
e \frac{\partial n_{\uparrow(\downarrow)}}{\partial t}=\frac{\partial j_{y, \uparrow(\downarrow)}}{\partial y}+\frac{e}{2 \tau_{s f}}\left(n_{\downarrow(\uparrow)}-n_{\uparrow(\downarrow)}\right), \\
j_{y, \uparrow(\downarrow)}=\sigma_{\uparrow(\downarrow)} E_{y}+e D \nabla n_{\uparrow(\downarrow)} \pm \gamma I_{x, \uparrow(\downarrow)},
\end{gathered}
$$

and

$$
\frac{\partial E_{y}}{\partial y}=\frac{e}{\varepsilon \varepsilon_{0}}[N(y)-n],
$$

where $-e$ is the electron charge, $n_{\uparrow(\downarrow)}$ is the density of spin-up (spin-down) electrons, $j_{y, \uparrow(\downarrow)}$ is the current density, $\tau_{s f}$ is the spin-relaxation time, $\sigma_{\uparrow(\downarrow)}=e n_{\uparrow(\downarrow)} \mu$ is the spin-up (spindown) conductivity, $\mu$ is the mobility, $D$ is the diffusion coefficient, $\epsilon$ is the permittivity of the bulk, and $\gamma$ is the parameter describing deflection of spin-up (+) and spindown (-) electrons. The current density $I_{x, \uparrow(\downarrow)}$ in $x$ direction is coupled to the homogeneous electric field $E(t)$ $=E_{0} \cos (\omega t)$ in the same direction as $I_{x, \uparrow(\downarrow)}=e n_{\uparrow(\downarrow)} \mu E(t)$. The last term in Eq. (2) is responsible for the spin Hall effect.

Equation (1) is the continuity relation that takes into account spin relaxation and Eq. (3) is the Poisson equation. Equation (2) is the expression for the current density in $y$ direction which includes drift, diffusion, and spin Hall effect components. We assume here for simplicity that $D, \mu, \tau_{s f}$, and $\gamma$ are equal for spin-up and spin-down electrons. ${ }^{13}$ In our model, as it follows from Eq. (2), the spin Hall correction to spin-up (spin-down) current densities [the last term in Eq. (2)] is simply proportional to the local spin-up (spin-down) density. All information about the microscopic mechanisms for the spin Hall effect is therefore lumped in the parameter $\gamma$.

Combining Eqs. (1) and (2) for different spin components we can get the following equations for the electron density $n=n_{\uparrow}+n_{\downarrow}$ and the spin-density imbalance $P=n_{\uparrow}-n_{\downarrow}$ :

$$
\frac{\partial n}{\partial t}=\frac{\partial}{\partial y}\left[\mu n E_{y}+D \frac{\partial n}{\partial y}+\gamma P \mu E(t)\right]
$$

and

$$
\frac{\partial P}{\partial t}=\frac{\partial}{\partial y}\left[\mu P E_{y}+D \frac{\partial P}{\partial y}+\gamma n \mu E(t)\right]-\frac{P}{\tau_{s f}} .
$$

\section{ANALYTICAL SOLUTION}

Before solving Eqs. (3)-(5) numerically, an instructive analytical result can be obtained in the specific case of exponential doping profile $N(y)=A \exp (\alpha y)$, with $\alpha$ a positive constant. At small values of $E_{0}$, we search for a solution in the form $n=n_{0}+\delta n, E_{y}=E_{y, 0}+\delta E_{y}, P=P_{0}+\delta P$. Setting $E_{0}$ $=0$, the leading terms in the above expansions can be easily obtained (see also Ref. 5): $n_{0}=N(y)=A e^{\alpha y}, E_{y}=-\frac{D \alpha}{\mu}, P_{0}=0$. Next, using Eq. (5) and neglecting the term $\sim \delta n E_{0}$, we obtain

$$
\delta P=\frac{e^{\alpha y} \gamma A \alpha \mu E_{0}}{\frac{1}{\tau_{s f}^{2}}+\omega^{2}}\left[\frac{1}{\tau_{s f}} \cos (\omega t)+\omega \sin (\omega t)\right] .
$$

Combining Eqs. (3), (4), and (6), integrating in $y$ and neglecting the term proportional to $\delta n \delta E_{y}$ (this approximation neglects small-amplitude higher harmonics terms), we obtain the following equation for $\delta E_{y}$

$$
\begin{aligned}
-\frac{\partial \delta E_{y}}{\partial t}= & \frac{e \mu A e^{\alpha y}}{\varepsilon \varepsilon_{0}} \delta E_{y}+\frac{\partial \delta E_{y}}{\partial y} D \alpha-\frac{\partial^{2} \delta E_{y}}{\partial y^{2}} D \\
& +\frac{e e^{\alpha y} \gamma^{2} A \alpha \mu^{2} E_{0}^{2}}{\varepsilon \varepsilon_{0}\left(\frac{1}{\tau_{s f}^{2}}+\omega^{2}\right)}\left[\frac{1}{\tau_{s f}} \frac{\cos (2 \omega t)+1}{2}+\omega \frac{\sin (2 \omega t)}{2}\right] .
\end{aligned}
$$

Equation (7) already demonstrates that the driving term for $\delta E_{y}$ involves a doubled frequency. In order to further proceed, let us consider a sample of a finite width $L$, in which doping level variations are not dramatic. Then, in the first term on the right-hand side of Eq. (7), we can write approximately $e^{\alpha y} \simeq e^{\alpha y^{*}}$, where $0<y^{*}<L$. This approximation allows us to find

$$
\delta E_{y}=\left[C_{1}+\sqrt{C_{2}^{2}+C_{3}^{2}} \cos (2 \omega t-\theta)\right] e^{\alpha y},
$$

where $\theta$, defined as $\tan \theta=C_{2} / C_{3}$, is a phase shift, and

$$
\begin{aligned}
& C_{1}=-\frac{G}{2} \frac{1}{\tau_{s f} \mu A e^{\alpha y^{*}}}, \quad G=\frac{\gamma^{2} A \alpha \mu^{2} E_{0}^{2}}{\frac{1}{\tau_{s f}^{2}}+\omega^{2}}, \\
& C_{2}=-\frac{G}{2} \frac{\frac{1}{\tau_{s f}}+\frac{e}{2 \varepsilon \varepsilon_{0}} \mu A e^{\alpha y^{*}}}{\frac{2 \varepsilon \varepsilon_{0} \omega}{e}+\frac{e}{2 \varepsilon \varepsilon_{0} \omega}\left(\mu A e^{\alpha y^{*}}\right)^{2}}, \\
& C_{3}=\frac{G}{2} \frac{\omega-\frac{1}{\tau_{s f}} \frac{e}{2 \varepsilon \varepsilon_{0} \omega} \mu A e^{\alpha y^{*}}}{\frac{2 \varepsilon \varepsilon_{0} \omega}{e}+\frac{e}{2 \varepsilon \varepsilon_{0} \omega}\left(\mu A e^{\alpha y^{*}}\right)^{2}} .
\end{aligned}
$$

Finally, the transverse voltage is given by

$$
V_{T}=-\int_{0}^{L} \delta E_{y} d y=\left[C_{1}+\sqrt{C_{2}^{2}+C_{3}^{2}} \cos (2 \omega t-\vartheta)\right] \frac{1-e^{\alpha L}}{\alpha} .
$$

Equation (12) demonstrates that there are two contributions to the transverse voltage $V_{T}$ : a shift term (proportional to $C_{1}$ ) 


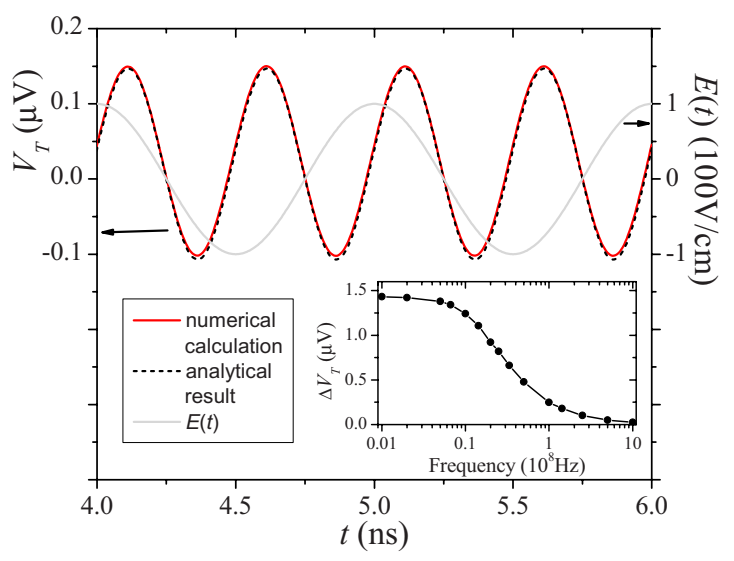

FIG. 2. (Color online) Frequency doubling in transverse voltage oscillations calculated at $\nu=10^{8} \mathrm{~Hz}$. A phase shift between the applied field $E(t)$ (gray solid line) and the transverse field $V_{T}$ (red solid line) oscillations is clearly seen. Inset: the transverse voltage oscillation amplitude as a function of the applied voltage frequency (the dots represent calculated values of $\Delta V_{T}$, the solid line is a fit to these points). The plots were obtained using the parameter values $\mu=8500 \mathrm{~cm}^{2} /(\mathrm{V} \mathrm{s}), D=55 \mathrm{~cm}^{2} / \mathrm{s}, \varepsilon=12.4, \tau_{s f}=10 \mathrm{~ns}, \gamma=10^{-3}$ (see Ref. 15), $E_{0}=100 \mathrm{~V} / \mathrm{cm}$ and the background density profile $N=10^{16} \exp (2 y / L) \mathrm{cm}^{-3}$, where $L=100 \mu \mathrm{m}$ is the sample width and $0 \leq y \leq L$. The analytical curve (black dashed line) was obtained at $y^{*}=L / 1.65$.

and a double-frequency phase-shifted oscillation term (proportional to $\sqrt{C_{2}^{2}+C_{3}^{2}}$ ). We have found that Eq. (12) is in excellent agreement with results of our numerical calculations (given below) with the only one adjustable parameter $y^{*}$. For a particular set of parameters used below, a perfect match between analytical and numerical calculations was obtained at $y^{*}=L / 1.65$.

\section{NUMERICAL SOLUTION}

Equations (3)-(5) can be solved numerically for any reasonable form of $N(y)$. We choose an exponential profile for its simplicity, the possibility to realize it in practice and for the purpose of comparison with the above analytical results. We solve these equations iteratively, starting with the electron density $n(y)$ close to $N(y)$ and $P(y)$ close to zero and recalculating $E_{y}(y)$ at each time step. ${ }^{14}$ At each time step, the transverse voltage is calculated as a change of the electrostatic potential across the sample.

Selected results of our numerical calculations are presented in Figs. 2 and 3. In particular, Fig. 2 demonstrates that the transverse voltage oscillations are indeed of a doubledfrequency character and phase-shifted with respect to the applied voltage. Another important feature shown in Fig. 2 is the excellent agreement between our analytical and numerical results. This agreement was obtained by an appropriate choice of the parameter $y^{*}$ defined after Eq. (7). We observed that $y^{*}$ slightly depends on $E_{0}$ and $\omega$, and, once $y^{*}$ is selected, the numerical and analytical solutions are in a good agreement in a wide range of excitation voltage parameters.

Multiple signatures of spin memristive behavior are clearly seen in Figs. 2 and 3 including a constant shift and phase shift in $V_{T}$ depicted in Fig. 2, frequency dependence of the transverse voltage oscillation amplitude shown in the inset of Fig. 2, and hysteresis behavior plotted in Fig. 3. All these features have a common origin: the adjustment of electron spin polarization to changing voltage takes some time. In particular, at low frequencies, we observe a small hysteresis in Fig. 3(a) because when the applied electric field is changed slowly (on $V_{T}$ equilibration time scale), at each moment of time $t$ the instantaneous $V_{T}$ is very close to its equilibrium value irrespective of the driving field $E(t)$. At high frequencies, the situation is opposite: when the applied electric field changes very fast, the electrons "experience" an average (close to zero) applied electric field, resulting in a significantly reduced transverse voltage oscillations amplitude.

We also note that at those moments of time when $E(t)$ $=0$, the transverse voltage is very close to, but not exactly, zero. This small deviation from an ideal memristive behavior ${ }^{9}$ (predicting $V_{T}=0$ when $E(t)=0$ and related to the absence of energy storage) should be a common feature of solid-state memristive systems operating at frequencies comparable to the inverse characteristic time of charge equilibration processes.

We conclude by noting that another way to realize the frequency doubling discussed in this paper can be realized by sandwiching a nonmagnetic homogeneous material between two ferromagnets (see schematic in Fig. 4). In this case one can employ the inverse spin Hall effect, in which the spin
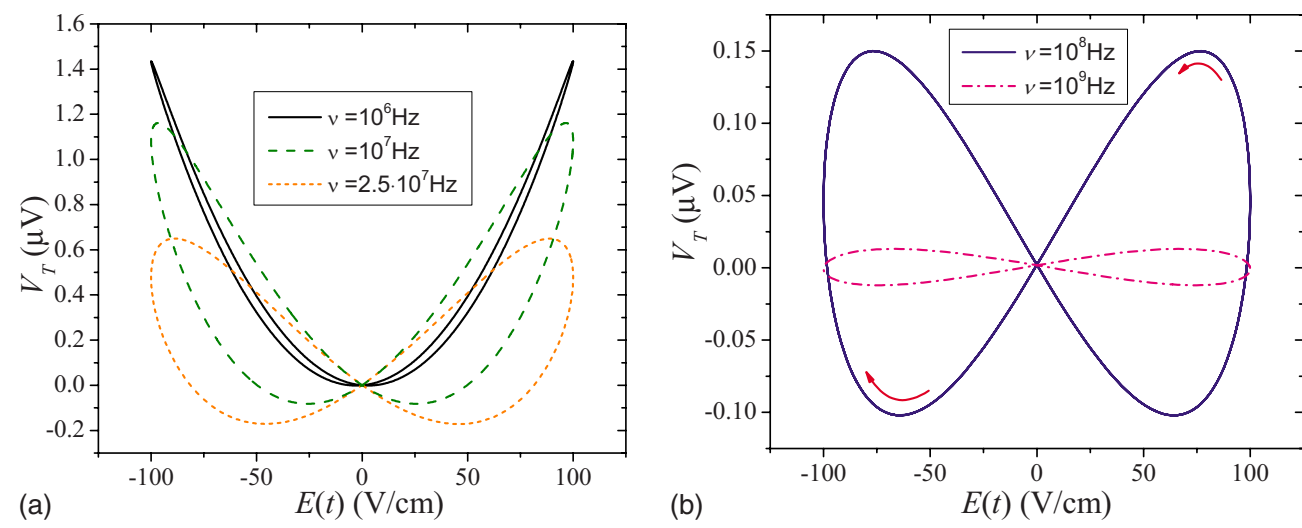

FIG. 3. (Color online) The transverse voltage as a function of applied electric field of (a) lower and (b) higher frequencies. 

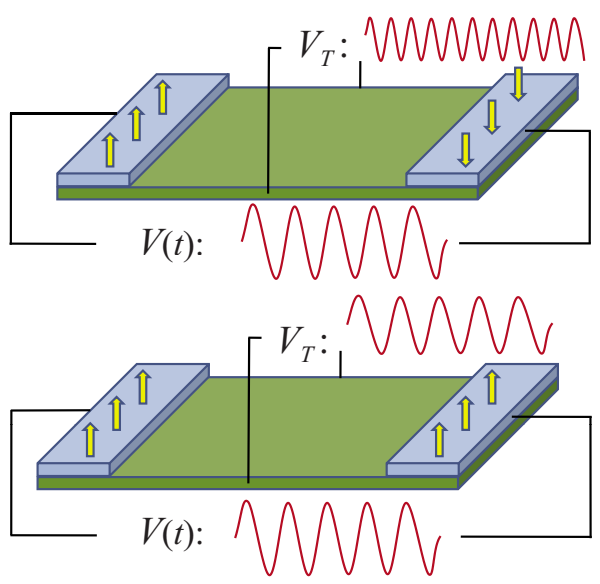

FIG. 4. (Color online) Frequency doubling with the inverse spin Hall effect where a nonmagnetic material (in the middle, green) is sandwiched between two ferromagnetic leads (at the ends, blue). The transverse voltage oscillation frequency depends on the relative orientation of the magnetization of the ferromagnetic contacts. The frequency is doubled when the direction of magnetization of ferromagnetic contacts is antiparallel. When the direction of magnetization of the ferromagnetic contacts is parallel, the transverse voltage frequency is equal to the applied voltage frequency. The oscillation amplitudes are not to scale.

current flowing in the nonmagnetic material induces transverse charge current, and thus causes charge accumulation. ${ }^{16-20}$ Since the electromotive force in the inverse spin
Hall effect is ${ }^{20} \sim \vec{J}_{S} \times \vec{\sigma}$, where $\vec{J}_{S}$ is the spin current along the sample and $\vec{\sigma}$ is the spin polarization, the simultaneous change of the current direction and its spin polarization, that occurs at antiparallel magnetization of ferromagnetic contacts, does not change the electromotive force polarity leading to transverse voltage oscillations with a doubled frequency. On the other hand, for parallel orientation of the spin polarization of the ferromagnetic contacts, the direction of spin current changes within each voltage cycle but not the direction of spin polarization. This thus results in a transverse voltage with the same frequency as the longitudinal one, albeit with lower amplitude (see Fig. 4). However, since spin injection allows for much higher levels of electron spin polarization (of the order of several tens of percents) compared to the spin Hall effect (normally, less than one percent), we expect the amplitude of $V_{T}$ oscillations to be larger in the inverse spin Hall effect. An external magnetic field can also be used in such experiments as an additional control parameter.

Finally, the phenomena we predict can be easily verified experimentally. They provide additional insight on the spin Hall effect and may find useful applications in electronics. We thus hope our work will motivate experiments in this direction.

\section{ACKNOWLEDGMENT}

This work has been partially funded by NSF Grant No. DMR-0802830. *pershin@physics.sc.edu

†diventra@physics.ucsd.edu

${ }^{1}$ P. A. Franken, A. E. Hill, C. W. Peters, and G. Weinreich, Phys. Rev. Lett. 7, 118 (1961).

${ }^{2}$ N. Bloembergen, Rev. Mod. Phys. 54, 685 (1982); C. Winterfeldt, C. Spielmann, and G. Gerber, ibid. 80, 117 (2008).

${ }^{3}$ K. W. Current and A. B. Current, Int. J. Electron. 45, 431 (1978).

${ }^{4}$ M. I. Dyakonov and V. I. Perel, JETP Lett. 13, 467 (1971); Phys. Lett. 35A, 459 (1971); J. E. Hirsch, Phys. Rev. Lett. 83, 1834 (1999).

${ }^{5}$ Yu. V. Pershin and M. Di Ventra, J. Phys.: Condens. Matter 20, 025204 (2008).

${ }^{6}$ D. B. Strukov, G. S. Snider, D. R. Stewart, and R. S. Williams, Nature (London) 453, 80 (2008).

${ }^{7}$ J. J. Yang, M. D. Pickett, X. Li, D. A. A. Ohlberg, D. R. Stewart, and R. S. Williams, Nat. Nanotechnol. 3, 429 (2008).

${ }^{8}$ Yu. V. Pershin and M. Di Ventra, Phys. Rev. B 78, 113309 (2008).

${ }^{9}$ L. O. Chua and S. M. Kang, Proc. IEEE 64, 209 (1976).

${ }^{10}$ M. I. Miah, Mater. Chem. Phys. 111, 419 (2008).

${ }^{11}$ Z. G. Yu and M. E. Flatté, Phys. Rev. B 66, 201202(R) (2002); W.-K. Tse, J. Fabian, I. Žutić, and S. Das Sarma, ibid. 72,
241303(R) (2005).

${ }^{12}$ Yu. V. Pershin and M. Di Ventra, Phys. Rev. B 75, 193301 (2007).

${ }^{13}$ This is a good approximation for the range of parameters considered in this work.

${ }^{14} \mathrm{We}$ have employed the Scharfetter-Gummel discretization scheme [D. L. Scharfetter and H. K. Gummel, IEEE. Trans. Electron. Devices 16, 64 (1969)] to solve both Eqs. (4) and (5) numerically.

${ }^{15}$ H.-A. Engel, B. I. Halperin, and E. I. Rashba, Phys. Rev. Lett. 95, 166605 (2005); H.-A. Engel, E. I. Rashba, and B. I. Halperin, ibid. 98, 036602 (2007).

${ }^{16}$ A. A. Bakun, B. P. Zakharchenya, A. A. Rogachev, M. N. Tkachuk, and V. G. Fleisher, JETP Lett. 40, 1293 (1984).

${ }^{17}$ H. Zhao, E. J. Loren, H. M. van Driel, and A. L. Smirl, Phys. Rev. Lett. 96, 246601 (2006).

${ }^{18}$ S. O. Valenzuela and M. Tinkham, Nature (London) 442, 176 (2006).

${ }^{19}$ T. Kimura, Y. Otani, T. Sato, S. Takahashi, and S. Maekawa, Phys. Rev. Lett. 98, 156601 (2007).

${ }^{20}$ K. Harii, K. Ando, K. Sasage, and E. Saitoh, Phys. Status Solidi C 4, 4437 (2007). 